\title{
NONLINEAR BEHAVIOR IN BRAY-LIBHAFSKY CHEMICAL REACTION
}

\author{
JIE REN, JINZHANG GAO*, JIE QU, XIAOXIA WEI, XIAODONG CHEN, WU YANG \\ (College of Chemistry and Chemical Engineering, Northwest Normal University, Lanzhou, 730070, P. R. China) \\ (Received: January 16, 2008 - Accepted: June 17, 2008)
}

\begin{abstract}
The Bray-Liebhafsky reaction exhibits different nonlinear behaviors during the iodate catalyzed decomposition of acidic hydrogen peroxide in the different conditions. Both the chaotic and regular oscillations were observed. The largest Lyapunov exponent $\left(\lambda_{\mathrm{L}}\right)$, the power spectrum and the $\log (\mathrm{P})-\log (\mathrm{f}) \operatorname{dependence}$ for power spectrum were used to evaluate these nonlinear behaviors. The effect of initial concentration of reactants on the types of oscillations and the initial potential $\left(\mathrm{E}_{\mathrm{o}}\right)$ were discussed in detail. The possible mechanism of the Bray-Liebhafsky reaction was also approached in this work.
\end{abstract}

Keywords: Bray-Liebhafsky reaction; largest Lyapunov exponent; power spectrum; mechanism

\section{INTRODUCTION}

The nonlinear chemical phenomena known as "oscillating chemical reaction" are complex dynamic systems that have so far been examined mainly in physico-chemical terms in order to elucidate the complex mechanism. Thus, various dynamics regimes including regular oscillations, periodic doubling, quasi-periodicity and deterministic chaos have been explored with a view to their characterization in recent years [1-4]. In general, a system exhibits different nonlinear phenomena because of the control parameters. Changing the control parameters may lead to the appearance of simple, single-peak periodic oscillations, complex multipeak periodic oscillations and chaotic dynamics. Transitions between these oscillations are caused by various bifurcations. So it is very important for experiments to identification of bifurcations, because it allows us to predict the value of control parameter at which appropriate type of oscillations or chaos occurs [5-6].

Several oscillating chemical systems, such as the Belousov-Zhabotinsky (B-Z) system, the $\mathrm{Cu}(\mathrm{II})$-catalysed reaction between hydrogen peroxide and sodium thiocyanate in alkaline medium, the Bray-Libhafsky (B-L) [7] and Briggs-Rauscher (B-R) $[8,9]$ systems have been studied. The most widely known and studied oscillating chemical is based on the $\mathrm{B}-\mathrm{Z}$ reaction, which involves the oxidation of an organic substrate by bromate ion in sulfuric acid medium. However, over 30 years before the discovery of B-Z oscillating reaction, Bray [7] observed oscillations during the B-L nonlinear system. The B-L oscillating chemical reaction involves the decomposition of hydrogen peroxide in the presence of iodate ion in acidic medium. The overall chemical reaction can be described simply by the following process:

$$
2 \mathrm{H}_{2} \mathrm{O}_{2}(\mathrm{aq}) \rightarrow 2 \mathrm{H}_{2} \mathrm{O}(\mathrm{aq})+\mathrm{O}_{2}(\mathrm{~g})
$$

Although the B-L reaction seems to be simpler than the B-Z system, it is not easy to describe the mechanism. Just due to the simple system having fewer variables and the detection of product (oxygen) being difficult, there have been few papers of the analysis of mechanism of such reaction [10, 11]. Matsuzaki [12] proposed a mechanism involving $\mathrm{I}_{3}^{-}$; the question is how to verify it by experiment. Schmitz and Rooze [13] assumed that the HOI should be oxidized at fictitious equilibrium state, meanwhile, Sharma and Noyes [11] considered further that free radical may be formed in the redox process. Up to now there is an open question.

For studying nonlinear chemical system, the following methods were often used to describe them: the largest Lyapunov exponent $\left(\lambda_{\mathrm{L}}\right)$, the time series curve, power spectrum and the signal calculated from power spectrum, phase diagram, Poincare mapping and so on $[14,15]$. The objective of the present paper is to describe the nonlinear phenomena of B-L oscillating system and discuss the effect concentration of reactants in detail.

\section{EXPERIMENTAL}

Chemicals

All chemicals were of analytical-reagent grade and used as received. The solution of hydrogen peroxide was made daily and standardized with the $\mathrm{KMnO}_{4}$ solution; and then kept in a black polyethylene bottle to avoid decomposition. Solution of $\mathrm{KIO}_{3}$ was freshly made just before the use and kept in refrigerator. Doubly distilled and deionized water was used throughout.

\section{Apparatus}

The oscillating assembly comprised a $50 \mathrm{ml}$ glass reaction vessel fitted with a Model CS-501 thermostat (Shanghai Pujiang Analytical Instrumental Factory, China) and a Model ML-902 magnetic stirrer (Shanghai Pujiang Analytical Instrumental Factory, China) for homogenization. A CHI 832 electrochemical analytical instrumental (Shanghai Chenhua Instrumental Company, China) was used to record the potential change. A Type 213 platinum electrode was used as working electrode, a Type 213 platinum electrode as the counter electrode and a Type 217 saturated calomel electrode as the reference electrode against which all potentials were reported.

\section{Procedure}

\section{Potentiometric measurements:}

Potentiometric measurements were performed in a closed thermostatregulated glass container equipped with a magnetic stirrer. The stirring rate was kept at $800 \mathrm{rpm}$. All of the experiments were performed at $303 \pm 0.05 \mathrm{~K}$. The reagents were respectively maintained as the following concentrations: $\mathrm{KIO}_{3}$, 0.05-0.3 M, $5 \mathrm{~mL} ; \mathrm{H}_{2} \mathrm{O}_{2}, 0.1-0.6 \mathrm{M}, 5 \mathrm{~mL} ; \mathrm{H}_{2} \mathrm{SO}_{4}, 0.2-1.0 \mathrm{M}, 5 \mathrm{~mL}$.

\section{RESULTS AND DISCUSSION}

\subsection{Results:}

The largest Lyapunov exponent $\left(\lambda_{L}\right)$ gives a quantitative measure for the chaotic regime. The value is defined as $[16,17]$

Where $\Delta L_{\mathrm{o} i}$ is the distance between nearby trajectories in the phase space

$$
\lambda_{L}=\lim _{N \rightarrow \infty} \frac{1}{N} \sum_{i=1}^{N} \frac{1}{t_{i}-t_{0 i}} \log _{2}\left(\frac{\Delta L_{i}}{\Delta L_{0 i}}\right)
$$

of a system at time $t_{\mathrm{oi}}$ and $\Delta L_{i}$ is the value at time $t_{i}$.

Due to the value of $\lambda_{L}$ characterizing the divergence of nearby trajectories in the phase space, a plenty of nonlinear behaviors (regular oscillation or chaos) in the system could be expressed. In general, when the value of $\lambda_{L}$ is larger than 0.01 , the behaviors of oscillating system approach to chaos. Figure 1 shows the nonlinear behaviors observed in the B-L chemical system, in which the values of $\lambda_{\mathrm{L}}$ are -0.000475 in Figure 1a and 0.038893 in Figure $1 \mathrm{~b}$, respectively. That is to say, the oscillating behavior of system is changing from regular oscillation to chaos. When the above results in Figure 1 were reexpressed by using power spectrum, the clear time series for chaos or periodic oscillations were obtained. That is to say, for a periodic oscillation, there are only limited frequencies in the power spectrum, whereas for chaos, there are incalculable frequencies. Over the main frequency $f_{o}$ the power spectrum is characterized by $\log (\mathrm{P})-\log (\mathrm{f})$. When using this relationship to characterize the results in Figure 2, the new profiles are showing in Figure 3, here the value of $\operatorname{tg}(\alpha)$ indicates the type of nonlinear behaviors (regular oscillation or chaos). Usually, when the value of $\operatorname{tg}(\alpha)$ approaches 1 , the oscillating behavior of system could be considered as the regular oscillation and when it less than 1 far away the behavior of system should be chaos. That is to say, when the angle is about $45^{\circ}$, the oscillating behavior of system could be defined as the regular oscillation and when it less than $45^{\circ}$ far away the behavior of system should be chaos. Distinctly, the results are consistent with the value of largest Lyapunov exponent $\left(\lambda_{\mathrm{L}}\right)$ and $\operatorname{tg}(\alpha)$. According to the value of largest Lyapunov exponent $\left(\lambda_{L}\right)$, the value of $\operatorname{tg}(\alpha)$ and the power spectrum, a conclusion can be drown that the regular oscillation and chaos could appear in the B-L nonlinear system under the differently initial concentrations of regents used. 

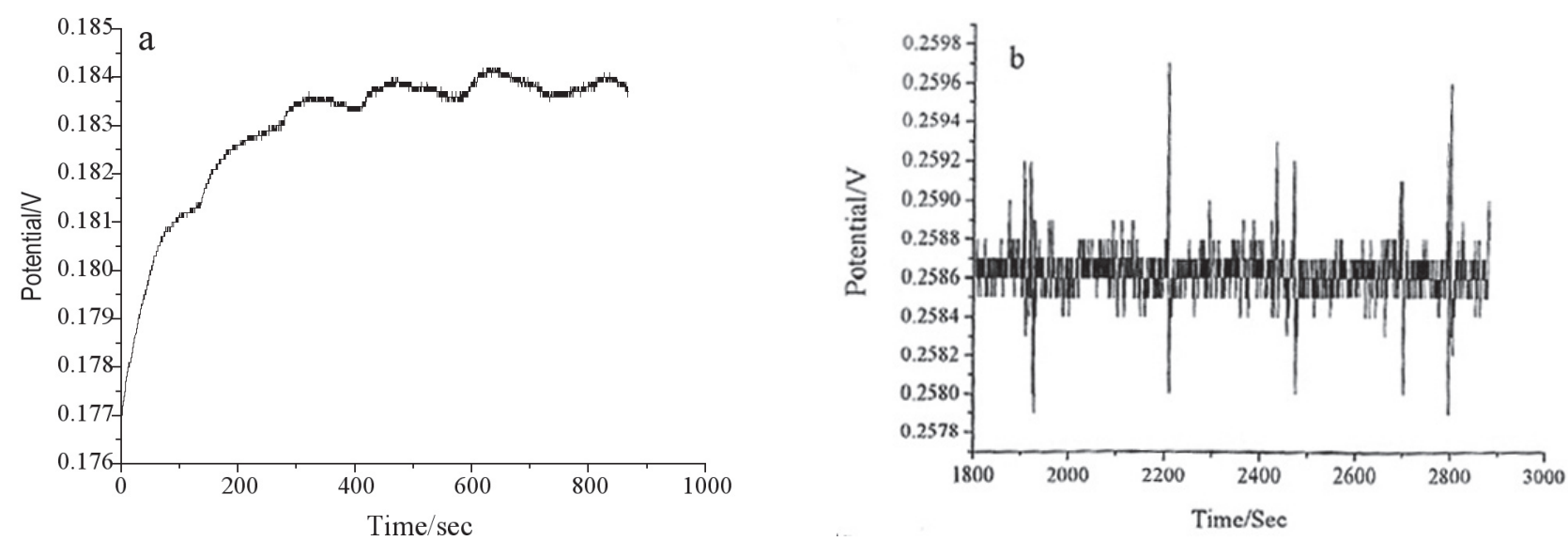

Figure 1. Time series observed in a batch reactor at the following initial concentrations of reagents.

a: $\left[\mathrm{H}_{2} \mathrm{O}_{2}\right]_{0}=0.2 \mathrm{M},\left[\mathrm{KIO}_{3}\right]_{0}=0.035 \mathrm{M},\left[\mathrm{H}_{2} \mathrm{SO}_{4}\right]_{0}=0.15 \mathrm{M} ; \mathrm{b}:\left[\mathrm{H}_{2} \mathrm{O}_{2}\right]_{0}=0.012 \mathrm{M},\left[\mathrm{KIO}_{3}\right]_{0}=0.1 \mathrm{M},\left[\mathrm{H}_{2} \mathrm{SO}_{4}\right]_{0}=0.3 \mathrm{M}$. common conditions: $\mathrm{T}=303 \mathrm{~K}, \mathrm{~S}(\mathrm{stirring}$ rate $)=800 \mathrm{rpm}$.
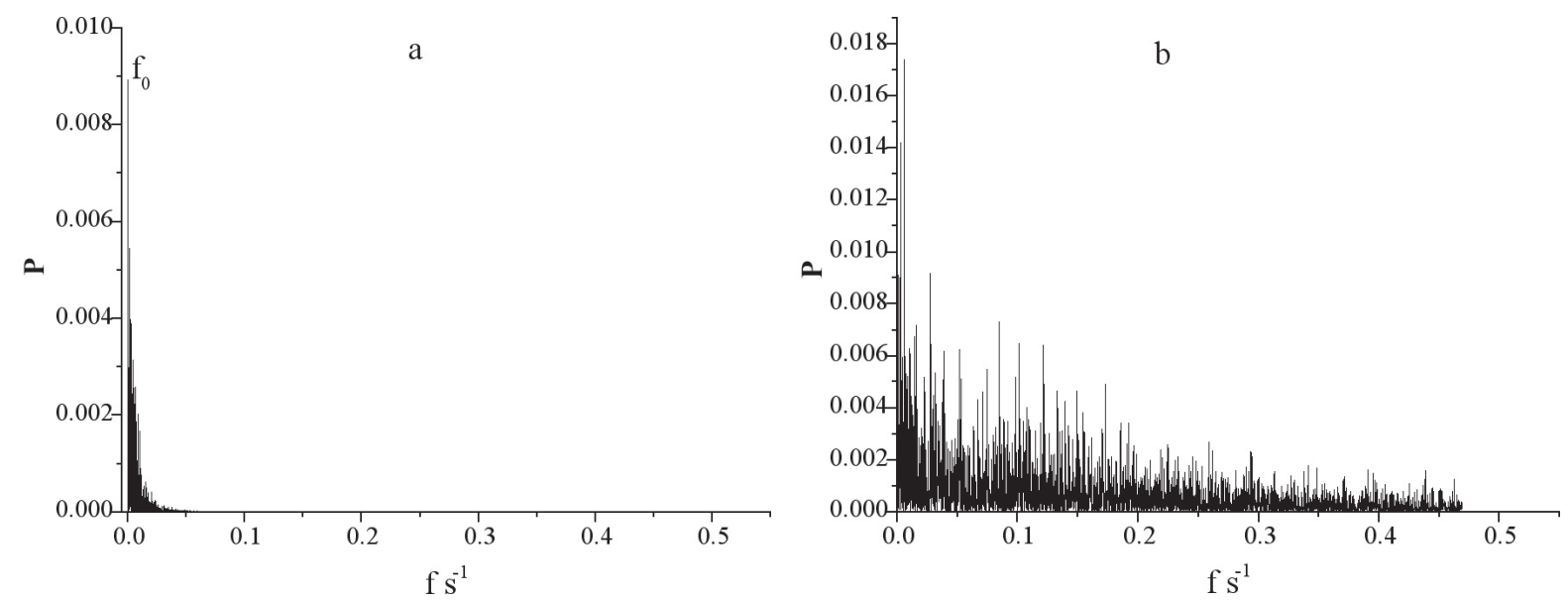

Figure 2. The power spectrum for the time series shown in Figure1. a: from Figure1a; b: from Figure1b.
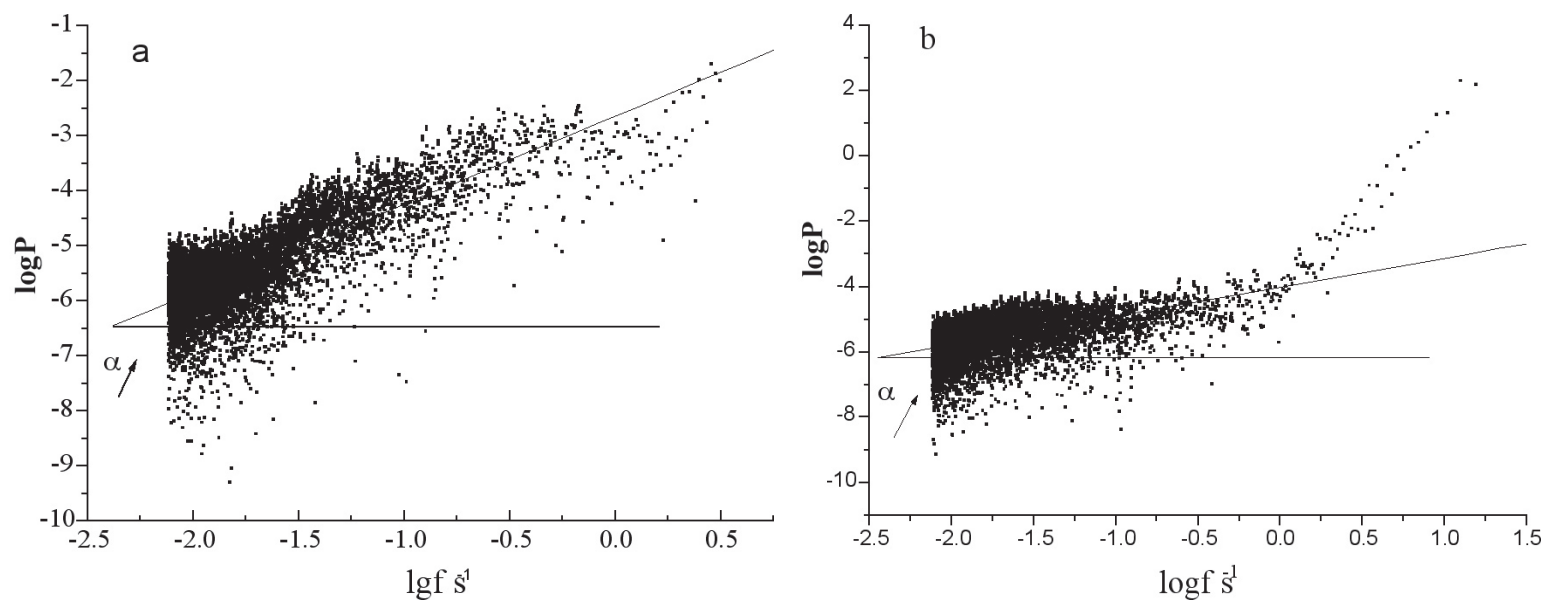

Figure 3. The $\log (\mathrm{P})-\log (\mathrm{f})$ dependence for power spectrum shown in Figure 2. $\mathrm{a}$ : from Figure 2a; $\mathrm{b}$ : from Figure $2 \mathrm{~b}$. 


\section{Discussion:}

Effect of hydrogen peroxide

The hydrogen peroxide is a key composition in B-L oscillating chemical system. Figure 4 shows the different time series at different concentrations of hydrogen peroxide. When the initial concentrations of $\mathrm{H}_{2} \mathrm{O}_{2}$ are at $0.03 \mathrm{M}$ and $0.1 \mathrm{M}$, the initial potential and its change trend are not similar to each other. If the concentration of hydrogen peroxide is chosen as the control parameter, the system exhibits various types of behavior. The dependence of the initial potential $\mathrm{E}_{0}$ on the initial concentration of $\mathrm{H}_{2} \mathrm{O}_{2}$ is shown in Figure $5 \mathrm{a}$. With increasing the initial concentration of $\mathrm{H}_{2} \mathrm{O}_{2}$, the initial potential $\mathrm{E}_{\mathrm{O}}$ decreases slightly. Bowers and Noyes [10] have pointed out that the dissolved oxygen is not a strong oxidizing agent. Even increasing the dissolved oxygen concentration more than 100 times, its oxidation potential increases only a 0.06 $\mathrm{V}$, and. Therefore, kinetic effects could be much more important part in this process.
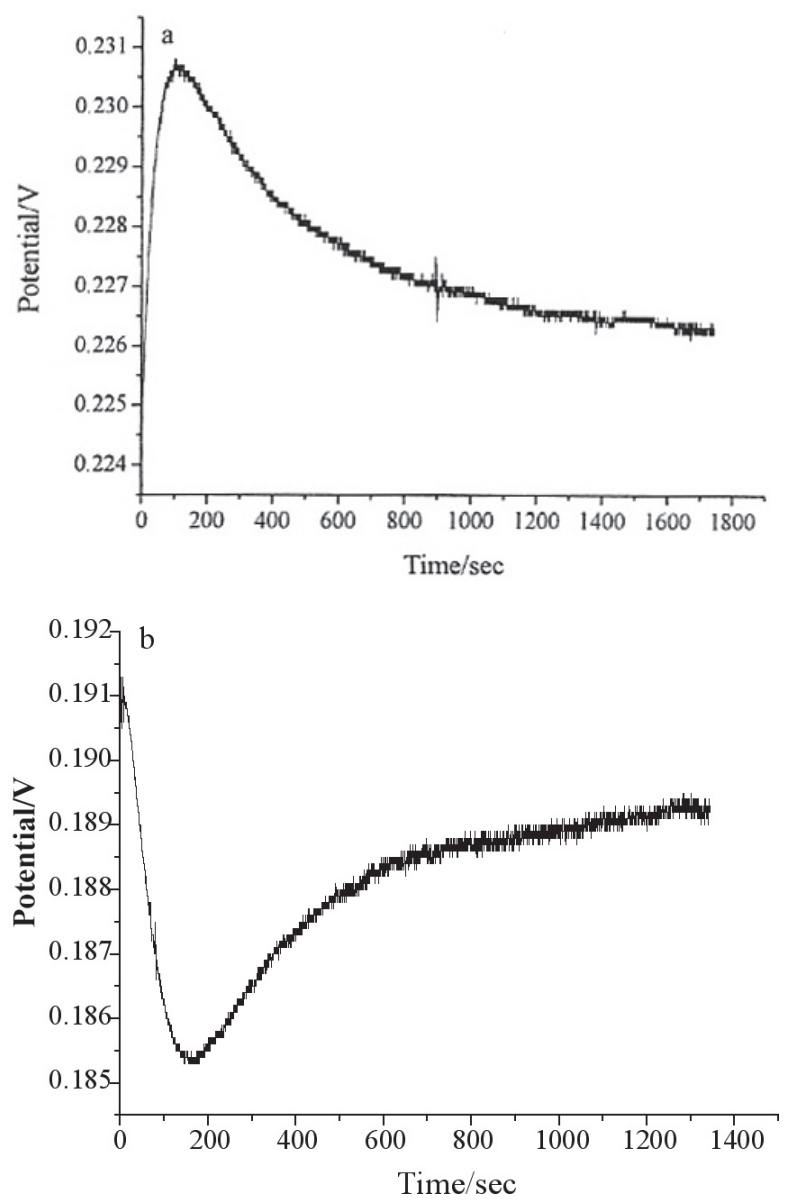

Figure 4. Time series observed in a batch reactor at different initial concentrations of hydrogen peroxide.

a: $\left[\mathrm{H}_{2} \mathrm{O}_{2}\right]_{0}=0.03 \mathrm{M} ; \quad$ b: $\left[\mathrm{H}_{2} \mathrm{O}_{2}\right]_{0}=0.1 \mathrm{M}$; common conditions: $\left[\mathrm{KIO}_{3}\right]_{0}=$ $0.1 \mathrm{M},\left[\mathrm{H}_{2} \mathrm{SO}_{4}\right]_{0}=0.3 \mathrm{M}, \mathrm{T}=303 \mathrm{~K}, \mathrm{~S}($ stirring rate $)=800 \mathrm{rpm}$.

\section{Effect of potassium iodate}

Figure $5 \mathrm{~b}$ shows the effect of potassium iodate on the initial potential $\mathrm{E}$. The effect of potassium iodate on the initial potential $\mathrm{E}_{\mathrm{o}}$ is unlike the effect of hydrogen peroxide completely. The initial potential increases with increasing the initial concentration, and the change for potassium iodate is larger than for hydrogen peroxide, because the absolute value of the slope in Figure $5 \mathrm{~b}$ is larger than that in Figure 5a.

Effect of sulfuric acid

Figure $5 \mathrm{c}$ shows the influence of sulfuric acid on the initial potential $\mathrm{E}_{\mathrm{o}}$. Although the initial potential increases with increasing the concentration sulfuric acid, the change is very slight. However, with decreasing acidity the period of oscillations is prolonged.
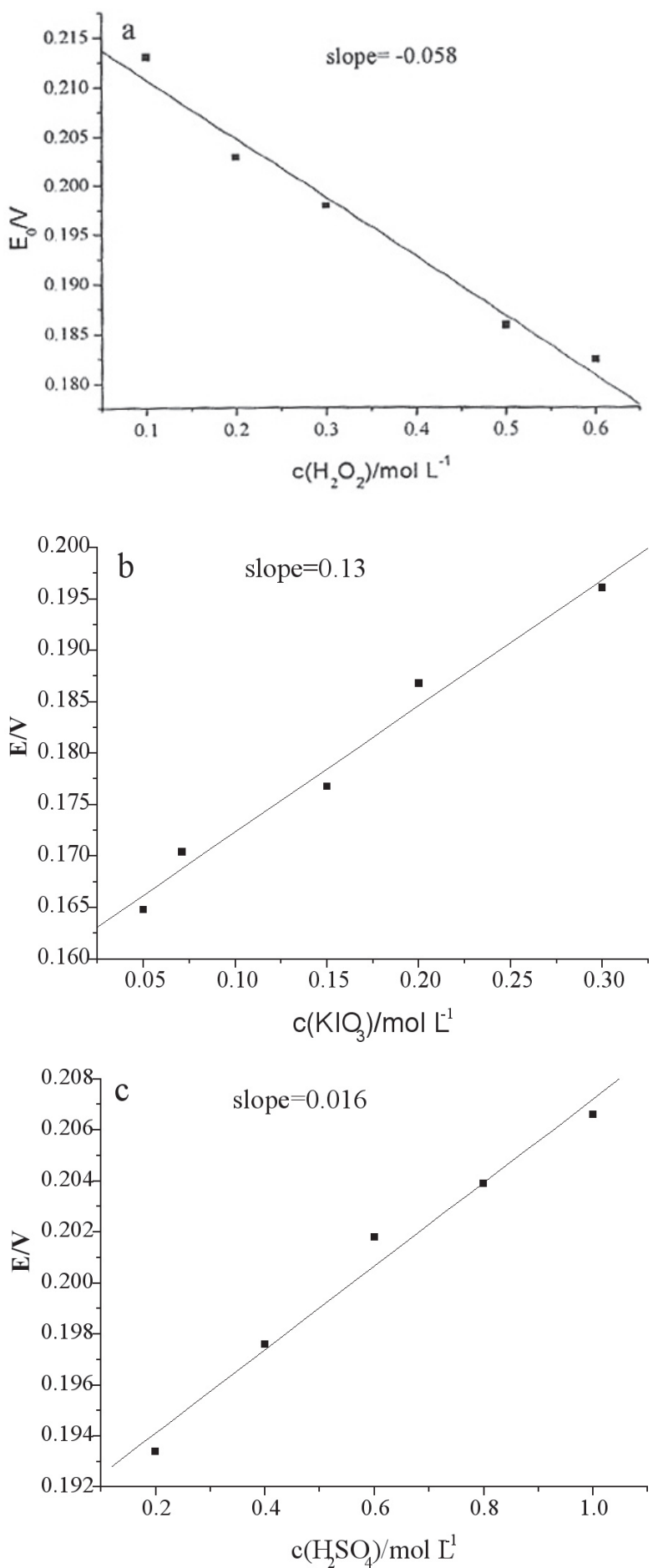

Figure 5. Relationship between the initial potential and the concentration of reactants.

$$
\text { a: } c\left(\mathrm{H}_{2} \mathrm{O}_{2}\right) \text { vs } \mathrm{E}_{\mathrm{o}} \text {; b: } \mathrm{c}\left(\mathrm{KIO}_{3}\right) \text { vs } \mathrm{E}_{\mathrm{o}} \text {; } \mathrm{c}: \mathrm{c}\left(\mathrm{H}_{2} \mathrm{SO}_{4}\right) \text { vs } \mathrm{E}_{\mathrm{o}} \text {. }
$$

The mechanism

The overall chemical reaction of B-L system can be described by the following steps: 
$2 \mathrm{H}_{2} \mathrm{O}_{2}(\mathrm{aq}) \rightarrow 2 \mathrm{H}_{2} \mathrm{O}(\mathrm{aq})+\mathrm{O}_{2}(\mathrm{~g})$

(A)

The reaction $(\mathrm{A})$ is assumed to be the net result of processes $(\mathrm{B})$ and $(\mathrm{C})$. $5 \mathrm{H}_{2} \mathrm{O}_{2}+2 \mathrm{IO}_{3}^{-}+2 \mathrm{H}^{+} \rightarrow \mathrm{I}_{2}+6 \mathrm{H}_{2} \mathrm{O}+5 \mathrm{O}_{2}$

$5 \mathrm{H}_{2} \mathrm{O}_{2}+\mathrm{I}_{2} \rightarrow 2 \mathrm{IO}_{3}^{-}+2 \mathrm{H}^{+}+4 \mathrm{H}_{2} \mathrm{O}$

(C)

The processes (B) and (C) alternately dominate the system, resulting in iodine oscillations and periodic oxygen gas evolution. In the B-L reaction, due to the oxygen producing continuously, a supersaturated solution containing oxygen would be formed. The dynamic influence of molecular oxygen on the system is becoming more important and the transport of oxygen from the solution to atmosphere will influence the overall reaction mechanism in the batch system. So the physical process of oxygen and iodine transport $[18,19]$ between the interphase should be supplemented in the overall reaction mechanism.

$$
\left\{\begin{array}{l}
\mathrm{O}_{2}(\mathrm{aq}) \rightarrow \mathrm{O}_{2}(\mathrm{~g}) \\
\mathrm{I}_{2}(\mathrm{aq}) \rightarrow \mathrm{I}_{2}(\mathrm{~g})
\end{array}\right\}
$$

Recently several papers reported the mechanism of B-L reaction [18-20], suggesting that the overall process can be divided into 10 individual steps, in which the transport of oxygen from the supersaturated solution into atmosphere may be a significant step. Noyes et al. [21] used this skeleton mechanism and proposed a model explored both analytically and numerically. Numerical integration for certain parameter values showed oscillations, but the model does not involve a true limited cycle. The 10 individual steps list below:

$$
\begin{aligned}
& \mathrm{I}^{-}+\mathrm{IO}_{3}^{-}+2 \mathrm{H}^{+} \rightarrow \mathrm{HIO}^{-} \mathrm{HIO}_{2} \\
& \mathrm{HIO}_{2}+\mathrm{I}^{-}+\mathrm{H}^{+} \rightarrow \mathrm{I}_{2} \mathrm{O}+\mathrm{H}_{2} \mathrm{O} \\
& \mathrm{I}_{2} \mathrm{O}+\mathrm{H}_{2} \mathrm{O} \rightarrow 2 \mathrm{HIO}_{2} \\
& \mathrm{HIO}+\mathrm{I}^{-}+\mathrm{H}^{+} \rightarrow \mathrm{I}_{2}+\mathrm{H}_{2} \mathrm{O} \\
& \mathrm{HIO}+\mathrm{H}_{2} \mathrm{O}_{2} \rightarrow \mathrm{I}^{-}+\mathrm{O}_{2}+\mathrm{H}^{+}+\mathrm{H}_{2} \mathrm{O} \\
& \mathrm{I}_{2} \mathrm{O}+\mathrm{H}_{2} \mathrm{O}_{2} \rightarrow \mathrm{HIO}^{-}+\mathrm{HIO}_{2} \\
& \mathrm{HIO}_{2}+\mathrm{H}_{2} \mathrm{O}_{2} \rightarrow \mathrm{IO}_{3}^{-}+\mathrm{H}^{+}+\mathrm{H}_{2} \mathrm{O} \\
& \mathrm{OO}_{3}^{-}+\mathrm{H}_{2} \mathrm{O}_{2}+\mathrm{H}^{+} \rightarrow \mathrm{HIO}_{2}+\mathrm{O}_{2}+\mathrm{H}_{2} \mathrm{O} \\
& \mathrm{O}_{2}(\mathrm{aq}) \rightarrow \mathrm{O}_{2}(\mathrm{~g}) \\
& \mathrm{I}_{2}(\mathrm{aq}) \rightarrow \mathrm{I}_{2}(\mathrm{~g})
\end{aligned}
$$

The positive cell potential suddenly rises, while the amount of oxygen evolved increases slowly at that time. Just after dropping suddenly the positive cell potential, the maximum of oxygen evolution is reached. If a drop of starch solution is added into the system at the minimum of positive cell potential, the color change is very little. With increasing the positive cell potential slowly, the speed of oxygen evolution decreases and the concentration of $I_{2}$ increases, so the color of system becomes deeper and deeper. When the positive cell potential increases upto the top point, the concentration of $I_{2}$ in the system reaches the maximum. This indicates that the maximum of oxygen evolution is reached and the iodine concentration in solution is minimal. In other words, oxygen evolution is greatest during that phase while $\mathrm{I}_{2}$ is oxidized. However, because the transport process in gases are much faster than in liquids, an excess of $\mathrm{O}_{2}$ in the gas phase will be easy to reach the equilibrium. If both gaseous and liquid phases are co-present, then supersaturated solution can exist for a longer time. At that time, many bubble nuclei will be created virtually. Those bubbles of oxygen will form rapidly and the equilibrium is approached [16]. Further investigations should be carried out to confirm the effect of supersaturated oxygen on the nonlinear behavior of system.

\section{CONCLUSIONS}

The experiments showed that in the different initial concentrations of reactants, the Bray-Liebhafsky reaction exhibited dissimilar nonlinear behaviors (periodic oscillations or chaos). The value of largest Lyapunov exponent $\left(\lambda_{L}\right)$, the time series curve, power spectrum and the signal calculated from power spectrum $\log (\mathrm{P})-\log (\mathrm{f})$ will be the useful methods to describe the nonlinear behaviors in the Bray-Liebhafsky reaction. The effects of hydrogen peroxide, potassium iodate and sulfuric acid were investigated respectively. The results showed that the concentration of hydrogen peroxide is the key parameter in the system. The transports both oxygen and iodine between the inter-phases are quite important, too.

\section{ACKNOWLEDGMENTS}

This work was supported by the Project of International Cooperation between China and Ukraine (043-05), the National Natural Science Foundation (20475044), the Project of KJCXGC-01, NWNU, and Gansu Key Lab of Polymer Materials, China.

\section{REFERENCE}

1. Field, R.J., Burger, M., Oscillations and travelling waves in chemical systems, Wiley, New York, 1985.

2. Strizhak, P.E., Ali, F., Menzinger, M., Stirring effect on bistability in a CSTR. 2. Theoretical analysis of the coalescence redispersion model for one-variable systems, J.Phys.Chem.A, 103, 10866 (1999).

3. Field, R.J., Schneider, F.W., Oscillating chemical reactions and nonlinear dynamics, J.Chem.Educ., 66, 195 (1989).

4. Kawczynski, A.L., Strizhak, P.E., Period adding and broken Farey tree sequence of bifurcations for mixed-mode oscillations and chaos in the simplest three-variable nonlinear system, J.Chem.Phys., 112 (14), 6122(2000).

5. Khavrus, V.O., Strizhak, P.E., Kawczynski, A.L., Scalings of mixedmode regimes in a simple polynomial three-variable model of nonlinear dynamical systems, Chaos, 13 (1), 112 (2003).

6. Kawczynski, A.L., Khavrus, V.O., Strizhak, P.E., Complex mixed-mode periodic and chaotic oscillations in a simple three-variable model of nonlinear system, Chaos, 10 (2), 299 (2000)-

7. Bray, W.C., A periodic reaction in homegenous solution and its relation to catalysis, J.Am.Chem.Soc., 43, 1262 (1921).

8. Furrow, S.D., Noyes, R.M., The Oscillatory Briggs-Rauscher Reaction. 1.Examination of Subsystems, J.Am.Chem.Soc., 04 (1), 38 (1982).

9. Furrow, S.D., Noyes, R.M., The Oscillatory Briggs-Rauscher Reaction. 2.Effect of Substitutions and Additions, J.Am.Chem.Soc., 104 (1),42 (1982).

10. Bowers, P.G., Hofstetter, Ch., Letter, C.R., Toomey, R.T., Suppersaturation limit for homogenous nucleation of oxygen bubbles in water at elevated pressure: "superhenry's law", J. Phys. Chem., 99, 9632 (1995).

11. Sharma, K.R., Noyes, R.M., Oscillation in chemical system: a detailed molecular mechanism for the Bray-Liebhafsky reaction of iodate and hydrogen peroxide, J.Am.Chem.Soc., 98 (15), 4345 (1976).

12. Treindl, L., Noyes, R.M., A new explanation of the oscillations in BrayLiebhafsky reaction, J.Phys.Chem., 97 (4), 11354 (1993).

13. Schmitz, G., Rooze, H., Far from equilibrum synergetics, Springer-Verlly, Berlin, 51 (1979).

14. Didenko, O.Z., Strizhak, P.E., Effect of temperature and small amounts of metal ions on transient chaos in the batch Belousov-Zhabotinsky system, Chem.Phys.Lett., 340, 55 (2001).

15. Goryachev, A., Strizhak, P.E., Kapral, R., Slow manifold structure and the emergence of mixed-mode oscillations, J.Chem.Phys., 107, 2881 (1997).

16. Strizhak, P.E., Didenko, O.Z., Ivashchenko, T.S., Determination of traces of thallium using the transient chaotic regime in the Belousov-Zhabotinskii oscillating chemical reaction, Anal.Chem.Acta., 428, 15 (2001).

17. Strizhak, P.E., Application of chemical chaos to analytical chemistry, Advances in Complex Systems, 6 (1), 1 (2003).

18. Sevcik, P., Kissimonova, K., Adamcikova, L., Oxygen production in the oscillatory Bray-Liebhafsky reaction, J.Phys.Chem.A., 104, 3958 (2000).

19. Valent, I., Adamcikova, L., Sevcik, P., Simulations of the iodine interphase transport effect on the oscillating Bray-Liebhafsky reaction, J. Phys. Chem., 102, 7576 (1998).

20. Kissimonova, K., Valent, I., Adamcikova, L., Sevcik, P., Numerical simulations of the oxygen production in the oscillating Bray-Liebhafsky reaction, Chem.Phys.Lett., 341, 345 (2001).

21. Noyes, R.M., Kalachev, L.V., Field, R.J., Mathematical model of the Bray-Liebhafsky oscillations, J. Phys. Chem., 99, 3514 (1995). 